\title{
Tubercular scleritis
}

\author{
Sharma R ${ }^{1}$, Marasini $\mathrm{S}^{2}$, Nepal BP ${ }^{3}$
}

${ }^{1-3}$ Department of Ophthalmology,

Kathmandu University Teaching Hospital, Kavre, Nepal

\section{Corresponding author}

Dr Ranjana Sharma

Department of Opthalmology

Kathmandu University Hospital

Email: ranjana_sharma66@yahoo.com

\begin{abstract}
Scleritis is a severe painful inflammatory process centred in the sclera. A scleritis may be a harbinger of many systemic diseases so that it is important to exclude multisystem disease. We describe a case of unilateral tubercular anterior nodular non-necrotizing scleritis. Anterior nodular non-necrotizing scleritis is relatively an uncommon manifestation of tuberculosis. It may be difficult to diagnose and manage the disease. However, good history, detail clinical evaluation, appropriate and timely management shows a good response to the disease.
\end{abstract}

Key Words

mantoux test, nodular scleritis, ocular tuberculosis

\section{INTRODUCTION}

Scleritis is typically a severe painful inflammatory process centred in the sclera which may involve the cornea, adjacent episclera, and underlying uvea. ${ }^{1}$ A scleritis may be a harbinger of systemic diseases like tuberculosis, Sarcoidosis, Behcet's disease, Rheumatoid Arthritis, other systemic immune mediated diseases ${ }^{2}$ and connective tissue diseases. ${ }^{3}$ Although scleritis is relatively uncommon, it is a potentially vision threatening condition.

Watson classified scleritis as anterior and posterior. Anterior scleritis has been further classified into diffuse, nodular, necrotising with inflammation and necrotising without inflammation and scleromalacia perforans. As scleritis can be the presenting feature of systemic diseases, it is important to exclude multisystem disease and treat it as it helps to control the ocular inflammation ${ }^{1}$.

About $45 \%$ of total population in Nepal is infected with tuberculosis4. Although Tuberculosis has been a rare cause of scleritis now a days, ${ }^{1}$ we describe a case of unilateral tubercular nodular non-necrotizing anterior scleritis.

\section{CASE REPORT}

A 30-year-old lady; lactating mother of two year old child, presented with severe uniocular pain, redness, and blurring of vision, excessive lacrimation and photophobia of her left eye since 26 days. She also had associated left sided headache and ear pain with nausea and lethargy. It was her first episode and was not preceded by trauma or any ocular surgery. She neither complained of any problem in her opposite eye nor gives history of any joint pain, dryness of mouth, fever or any other systemic illness. There was no history of use of any systemic or topical drugs prior to the illness. She does not give history of tuberculosis either in family or in neighbourhood. She previously was evaluated and treated in a private clinic and then finally referred to our hospital for further evaluation and management, as there was no sign of improvement. She was on the Prednisolone (1\%) eye drops four times a day, Atropine (1\%) eye drops two times a day and Ofloxacin eye drops $(0.3 \%)$ four times a day since three weeks.

On examination she was in severe pain, however, she was conscious, cooperative and well oriented to time, place and person. She was thinly built and her weight was $40 \mathrm{~kg}$ and height- of $150 \mathrm{~cm}$. Her vital parameters were within normal limits including temperature of $37^{\circ}$ Centigrade. Systemic examination including respiratory and musculoskeletal system was reported normal by medical internist on consultation. 
Her visual acuities were 6/6 right and 6/12 left unaided. Lids and adnexa were normal however blepharospasm was present in left eye. Sectoral conjunctival congestion in nasal side from 7-9 'o' Clock position with circum-corneal congestion was present. Episcleral vessels were engorged. There was $6 \mathrm{~mm} \times 8 \mathrm{~mm}$ yellowish single nodule on inferonasal quadrant at 8 o'clock position of sclera with overlying and surrounding oedema. Pupil was pharmacologically dilated. No abnormality was there in cornea, iris, anterior chamber, as well as in posterior segment. Opposite eye was normal. Intraocular pressure (Goldman Tonometer) was $16 \mathrm{mmHg}$ right and $12 \mathrm{mmHg}$ left eye respectively.

On the basis of these features, left eye anterior nodular non necrotizing scleritis was diagnosed. She was advised for radiological investigation, lab investigation and internist consultation to rule out any systemic diseases. Meantime Prednisolone (1\%) eye drop 4 times a day, Atropine (1\%) eye drops three times a day, Ofloxacin (0.3\%) eye drops three times a day, were continued and tablet Indomethacin $(25 \mathrm{mg})$ three times a day, and Capsule

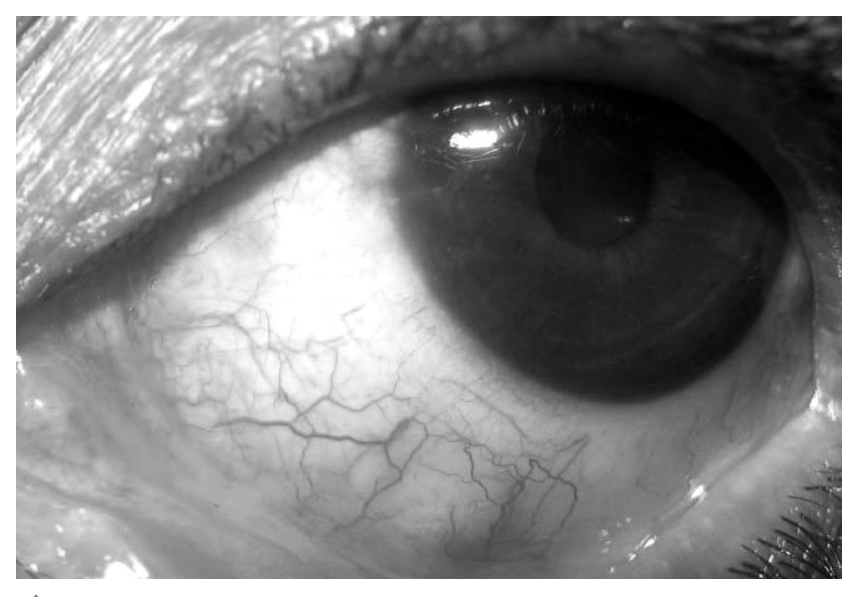

Figure 1. Scleral nodule at presentation

Pantoprazole $(40 \mathrm{mg})$ once a day was prescribed. She was asked to follow up with lab reports. Chest X-ray was reported to be normal. Her total Count (TC) was 6000 cumm with Neutrophil $=71 \%$, Leucocyte $=25 \%$, Basophil $=2 \%$, Eosinophil $=2 \%$ and $\mathrm{ESR}=27 \mathrm{~mm} / \mathrm{Hr}$ (Wintrobe method). C- reactive protein was positive. In Mantoux test, $18 \mathrm{~mm}$ induration diameter was seen after 72 hours.

In view of normal blood and radiological investigations, sputum negative for Acid Fast Bacilli (AFB) bacilli, higher reading of Mantoux test, unresponsive to the topical steroid and high endemicity of tuberculosis in the country 4 ; a diagnosis of the tubercular scleritis was made.

Anti-Tubercular Therapy (ATT), Isoniazid(H), Rifampicin(R), Pyrazinamide, Ethambutol plus four months of Isoniazid and Rifampicin (dose calculated according to her body weight) was started after patient screened for any renal and hepatic abnormalities. Along with ATT topical steroid and atropine were also continued. Oral steroid $1 \mathrm{mg} / \mathrm{kg}$ body weight was added after 10 days of starting ATT, which was tapered after 2 weeks over 1 month.

Patient showed dramatic response within one week of starting treatment as her pain and redness decreased to minimal. Nodule started resolving after 3 weeks of treatment. Presently patient is on regular follow-up with no ocular or systemic complication of ATT and topical steroid.

\section{DISCUSSION}

Tuberculosis remains the world's leading infectious cause of death and causes a variety of diseases throughout the body and eye. Recent estimates are that 8-10 million new tuberculosis cases occur each year in the world and 2-3 million die $^{5}$. The demo-graphics of infection vary widely, with developing countries bearing the heaviest burden of disease.

Persons breathing in the Mycobacterium tuberculosis contaminated air can become infected asymptomatically with latent phase of the disease which may progress to tuberculosis in compromised immune system. It is estimated that one-third of the world's population has latent tuberculosis. ${ }^{6}$

Among scleritis anterior scleritis is the most common pattern of the disease. Patients with scleritis may present either with an underlying related disorder or in the absence of any known underlying systemic disease and it may result in severe ocular morbidity. The incidence of systemic disease in patients with scleritis is reported between 39$50 \%{ }^{2,3}$

Ocular tuberculosis has always been considered rare, yet its incidence has varied widely across time, patient populations, and geography. ${ }^{6}$ McCluskey PJ7 reported that patients older than age 50 are prone to have an increased risk of having an associated systemic disease and more likely to experience visual loss.

In one of the studies carried out in Spain by Bouza E et al. ${ }^{8}$ ocular tuberculosis was found in $18 \%$ of the 100 culture proven tuberculosis, important ocular findings being choroiditis, and other ocular lesions including papillitis, retinitis, vitritis, vasculitis, dacryoadenitis, and scleritis. Donahue ${ }^{9}$ found $1.4 \%$ ocular TB in 10, 000 patients with primary pulmonary $\mathrm{TB}$ who were evaluated in the eye clinic of the Mattapan Sanatorium, in Boston, Massachusetts, USA. In a study recently reported at Srilanka ${ }^{10}$, of the total 2130 tuberculosis patients, ocular tuberculosis was diagnosed in 23 patients among whom episcleritis and 


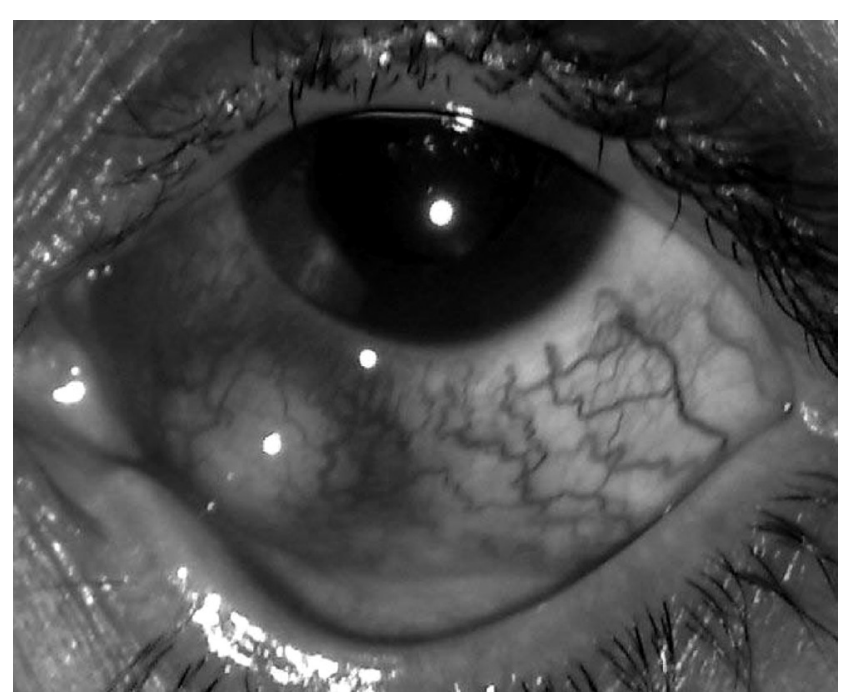

Figure 2. Five weeks after starting ATT

inflammatory scleral nodule were observed in one patient each.

The characteristic feature of scleritis is the severe pain that may involve the eye and orbit and radiates to involve the ear, scalp, face, and jaw. Scleritic pain is typically dull and boring in nature, often resistant to mild analgesics. There may be photophobia and lacrimation as well. Nodular anterior scleritis is characterized by a more localized area of scleral oedema such that distinct nodules result. They may be single or multiple nodules. There is no evidence of capillary closure or non-perfusion and no evidence of scleral necrosis. ${ }^{1}$

Infectious scleritis can be viral, bacterial, fungal and parasitic. The mechanism of inflammation in many infections is thought to be partly or wholly immune mediated. Infections occur in tissues compromised by disease or trauma and both iatrogenically and accidentally.

The analysis of the inflammatory cellular infiltrate in six episcleral biopsies and two enucleated eyes, it was seen that the inflammatory cells mainly involved were $\mathrm{T}$ lymphocytes and macrophages with a predominance of CD4 positive cells and few lymphocytes. In circumscribed areas neutrophils, macrophages, and plasma cells were part of the scleral infiltrate. It is therefore assumed that vascular or perivascular disease plays a role in the pathogenesis of scleral inflammation. Immune-mediated ocular TB can occur due to hypersensitivity to M. tuberculosis antigens from a distant focus (such as lungs). This hypersensitivity causes inflammation despite the absence of the bacterium in the eye. ${ }^{6,11}$

The lack of any uniform diagnostic criteria for intraocular tuberculosis, in either immune-competent or immunecompromised individuals, has contributed to the confusion regarding diagnosis and management. ${ }^{12,13}$
There is no pathognomonic ophthalmic finding for ocular TB. Clinician suspicion is an imperative first step toward the correct diagnosis. ${ }^{5-6}$ A detailed history of the patient is very important, to suspect ocular tuberculosis. The past history or medical history, family history or any contact with TB patients is important to rule out the disease.

Diagnosis of ocular tuberculosis is usually based on the positive Mantoux test, the caseating granulomatous inflammatory lesion on histopathology, the positive culture for mycobacterium tuberculosis if the specimens are obtained early in the course of the disease; and completes resolution of the disease with specific antituberculosis treatment. ${ }^{13-15}$ It has been specified that the Mantoux test showing induration of less than $5 \mathrm{~mm}$ in diameter does not rule out the possibility of ocular tuberculosis. Moreover, it is generally accepted that induration of $10 \mathrm{~mm}$ or more than $18 \mathrm{~mm}$ indicates a positive Mantoux test. ${ }^{16}$

Thompson $\mathrm{MJ}$ et $\mathrm{al}^{17}$ insist that an initial workup that yields negative results should not eliminate tuberculosis from the differential Diagnosis. They noted that tuberculous scleritis should be considered in patients' cases in which scleritis is unresponsive to traditional therapy and therapeutic trial with ant tuberculosis drugs can be an important practical way of diagnosing ocular tuberculosis, especially in places where various laboratory facilities are unavailable or unaffordable.

Management of ocular tuberculosis is a challenge in ophthalmic practice because it is extremely difficult to diagnose. Patients with scleritis need to have the type and extent of their disease diagnosed, the complications present detected and any underlying systemic or local cause defined. The aim of treatment is to remove or treat the cause where possible but in the majority is to control the inflammatory process to relieve the pain and thereby reduce the damage to the eye ${ }^{18}$. Some authors have emphasized the Isoniazid therapeutic trial in every case of uveitis with a positive tuberculin skin test of any size or strength ${ }^{19}$ while others ${ }^{20}$ have reported that the use of Isoniazid alone for diagnostic purposes is no better than a placebo. Therefore, a complete regimen of the treatment should be given to determine the therapeutic response.

Any scleritis that is associated with a systemic disease also usually requires more aggressive immunosuppressive therapy. ${ }^{7}$ Non-necrotizing scleritis is reported to readily respond to systemic NSAIDS. ${ }^{21}$ Corticosteroids are usually reserved for patients who do not respond to cox-inhibitors or those with posterior or necrotizing disease. ${ }^{21-22}$ A starting dose of $1 \mathrm{mg} / \mathrm{kg} /$ day is standard with weekly reduction by 20-25 mg/week until a dose of $40 \mathrm{mg} /$ day is reached. 
After this dose is reached, the rate of reduction is individualized, according to the clinical findings and patients' response, but is in the order of $5 \mathrm{mg}$ /week until cessation or an acceptable maintenance dose is reached. ${ }^{8,21}$ Both systemic administration and orbital floor and sub-Conjunctival injections have been successful although local steroid injections are less commonly used..$^{23}$ In a recently reported case, Roufas $\mathrm{A}$ et al. ${ }^{23}$ have noted that subconjunctival triamcilone therapy is an effective treatment with a prolonged duration of effect in selected patients with non-necrotizing scleritis that is refractory to the initial therapy.

Drug therapy for ocular tuberculosis (OTB) is similar to that for pulmonary tuberculosis. Anti-tubercular therapy (ATT) is used systemically in the treatment of OTB because it offers the best penetration of posterior ocular structures with least toxicity. Current guidelines recommend a sixmonth course of daily Isoniazid $(5 \mathrm{mg} / \mathrm{kg})$ and Rifampicin $(10 \mathrm{mg} / \mathrm{kg})$, in addition to Pyrazinamide $(15-30 \mathrm{mg} / \mathrm{kg})$ and Ethambutol $(15 \mathrm{mg} / \mathrm{kg})$ for the first two months. The therapy will also target other undiagnosed foci of disease which may co-exist. ${ }^{24}$

\section{CONCLUSION}

Anterior nodular non-necrotizing scleritis is a rare cause of tuberculosis. It may be very difficult to diagnose and manage once diagnosed. However good history, detail clinical evaluation, appropriate and timely management shows a good response to the disease. 


\section{REFERENCES}

1. Okhravi N, Odufuwa B, McCluskey P et al. Scleritis. Survey of ophthalmology 2005;50:4.

2. Sainz de la Maza M, Foster CS, Jabbur NS. Scleritis associated with rheumatoid arthritis and with other systemic immunemediated diseases. Ophthalmology 1994;101:1281-6.

3. A J Lyne and D A Pitkeathly. Episcleritis and scleritis. Their association with connective tissue disease. Ann Rheum Dis 1970;29:191-2.

4. National Tuberculosis Control programme, Annual Report. Nepal: [Nepal Tuberculosis Center]; 2002-03.

5. Kochi A. The global tuberculosis situation and the new control strategy of the World Health Organization. Tubercle 1991;72:1-6.

6. Tabbara KF. Ocular tuberculosis: anterior segment. Int Ophthalmol Clin 2005;45:57-69.

7. McCluskey PJ, Watson PG, Lightman S, et al. Posterior scleritis: clinical features, systemic associations, and outcome in a large series of patients. Ophthalmology 1999;106:2380-6.

8. Bouza E, Merino P, Muñoz P, et al. Ocular tuberculosis. A prospective study in a general hospital. Medicine (Baltimore) 1997;76:53-61.

9. Donahue HC. Ophthalmologic experience in a tuberculosis sanatorium. Am J Ophthalmol 1967;64:742-8.

10. BMGD Yasaratne, D Madegedara, N S Senanayake et al. A case series of symptomatic ocular tuberculosis and the response to anti-tubercular therapy. Ceylon Medical Journal 2010;55:1.

11. Kurup SK, Chan CC. Mycobacterium-related ocular inflammatory disease: diagnosis and management. Ann Acad Med Singapore 2006;35:203-9.

12. Gupta V, Gupta A, Rao NA. Intraocular tuberculosis-an update. Survey of ophthalmology 2007;52(6).
13. Sainz de la Maza M, Jabbur NS, Foster CS. Severity of scleritis and episcleritis. Ophthalmology 1994;101:389-96.

14. FongLP, Sainz dela Maza M, RiceBA, etal:Immunopathology of scleritis. Ophthalmology 1991;98:472-9.

15. Helm CJ, Holland GN. Ocular tuberculosis. Surv Ophthalmol 1993;38:229-56.

16. Carolyn TB, Elizabeth A, Talbot, et al. Diagnosis of Ocular Tuberculosis: A Role for New Testing Modalities? International Ophthalmology Clinics 2007;47:45-62.

17. Thompson MJ, Albert DM. Ocular Tuberculosis. Arch ophthalmol 2005:123; 844-9.

18. McCluskey P, Wakefield D: Current concepts in the management of scleritis. Aust NZJ Ophthalmol 1988;16:16976.

19. Abrams J, Schlaegel TF Jr. The role of the isoniazid therapeutic test in tuberculous uveitis. Am J Ophthalmol 1982;94:511-5.

20. Oksala A, Salminen A: Leptospiral uveitis. Acta Aphthalmol 1956;34:185-194.

21. Jabs DA, Mudun A, Dunn JP, et al. Episcleritis and scleritis: clinical features and treatment results. Am J Ophthalmol 2000;130:469-76.

22. Watson PG, Lobascher D. The diagnosis and management of episcleritis and scleritis. Trans Ophthalmol Soc UK 1965;85:369-78.

23. Roufas A, Jalaludin B, Gaskin C, et al. Subconjunctival triamcilone treatment for non-necrotizing scleritis. $\mathrm{Br} J$ ophthalmol [Internet]. 2010 May [cited Jan 2011]. Available from: http://bjo.bmj.com/content/early/2010/04/16/ bjo.2009.164962.full.pdf

24. National programme for tuberculosis control and chest diseases. General manual for tuberculosis control. 2nd ed. Ministry of Health, Sri Lanka: 2005. 